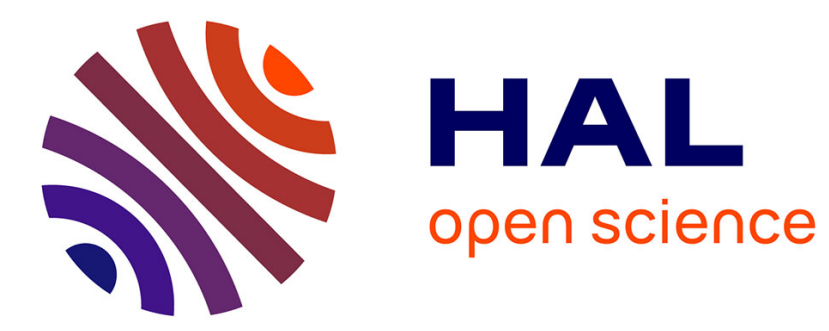

\title{
MATÉRIAUX ABSORBANTS SÉLECTIFS OBTENUS PAR IMPLANTATION IONIQUE
}

\author{
G. Abouchacra, G. Chassagne, A. Delmas
}

\section{To cite this version:}

G. Abouchacra, G. Chassagne, A. Delmas. MATÉRIAUX ABSORBANTS SÉLECTIFS OBTENUS PAR IMPLANTATION IONIQUE. Journal de Physique Colloques, 1981, 42 (C1), pp.C1-327-C1-337. 10.1051/jphyscol:1981123 . jpa-00220673

\section{HAL Id: jpa-00220673 https://hal.science/jpa-00220673}

Submitted on 1 Jan 1981

HAL is a multi-disciplinary open access archive for the deposit and dissemination of scientific research documents, whether they are published or not. The documents may come from teaching and research institutions in France or abroad, or from public or private research centers.
L'archive ouverte pluridisciplinaire HAL, est destinée au dépôt et à la diffusion de documents scientifiques de niveau recherche, publiés ou non, émanant des établissements d'enseignement et de recherche français ou étrangers, des laboratoires publics ou privés. 


\title{
MATÉRIAUX ABSORBANTS SÉLECTIFS OBTENUS PAR IMPLANTATION IONIQUE
}

\author{
G. Abouchacra, G. Chassagne et A. Delmas \\ Département de Physique des Matériaux, Université Claude Bernard, Lyon I, \\ 43 Boulevard du 11 Novembre 1918, 69622 Villeurbanne Cédex, France
}

1. Introduction.- Par implantation ionique dans un matériau diélectrique, transparent en l'occurence, les propriétés physiques peuvent être modifiêes. En effet, après un traitement thermique convenable, les ions métalliques implantés peuvent soit précipiter sous forme d'agrégats métalliques, soit donner ure phase nouvelle résultant d'interactions chimiques $/ 1 /$. Selon l'énergie d'implantation les ions pénètrent plus ou moins profondêment et se distribuent selon un profil de concentration d'allure gaussienne (Fig. 1).

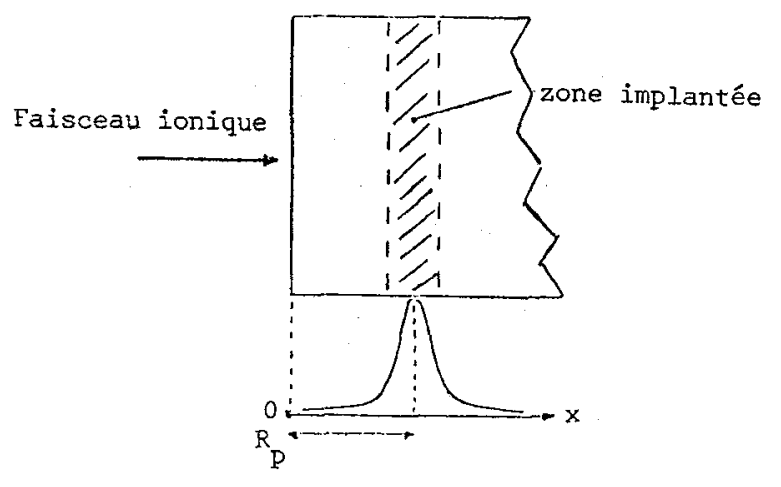

Fig. 1.- profil de concentration des ions implantés dans un matériau. $\mathrm{Rp}$ : profondeur d'implantation mesurée à la concentration maximale.

Des méthodes de caractérisation bien choisies permettent de définir ce profil et la nature et la structure de la phase obtenue par implantation.

Notre intérêt s'est porté sur la modification des propriétés optiques d'un oxyde ionique refractaire (MgO) implanté avec des ions métalliques présentant un caractère métallique pur (Na) ou un caractère métallique de transition $(A u)$, bien défini. Nous avons observé une forte absorption optique dans le domaine visible du spectre solaire /2/. 
2. But de l'étude.- Les matériaux sélectifs utilisés pour les absorbeurs des différents capteurs solaires doivent présenter une stabilité thermique de leur propriétess optiques en fonction du temps, et ceci dans la gamme d'utilisation de températures envisagées. Notre étude a porté sur des matériaux utilisables dans la gamme $300-500^{\circ} \mathrm{C}$, où l'association surfaces sélectives et concentration se trouve efficace, mais où la tenue thermique (đégradation) des matériaux sélectifs est très préoccupante.

Les matériaux composites de type cermet peuvent être bien adaptés à cette utilisation, à condition de bien choisir le matériau matrice ( $M g 0, \mathrm{Al}_{2} \mathrm{O}_{3}$ ) /3/. Par implantation métallique ( $\mathrm{Na}, \mathrm{Au}$ ) il est possible de réaliser un tel cermet, en espérant contrôler la fraction volumique : en effet, un cermet ou dispersion de particules métalliques dans une matrice diélectrique (céramique), voit se modifier ses propriétés en passant du comportement métallique au comportement diélectrique, en modifiant la fraction volumique. En principe on peut ainsi élaborer un matériau avec des propriêtês optiques désirées.

Le cermet ainsi développé doit se prêter à une caractérisation bien définie et permettre une meilleure compréhension des mécanismes de l'absorption sélective, en modélisant en fonction de la composition.

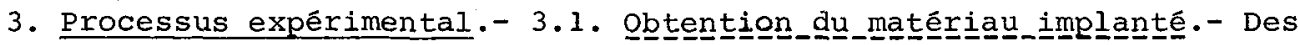
monocristaux (dont la caractêrisation sera plus simple) d'oxyde de magnésium Mgo de pureté $4 \mathrm{~N}$, de provenance $W$ et $C$ spicer Co., sont clivés selon (100). Les échantillons obtenus, d'environ 0,5 mm d'épaisseur, sont implantés avec des ions métalliques en utilisant un accélérateur de particules. Pour le sodium les énergies d'implantation choisies sont de 600 et $750 \mathrm{keV}$ afin d'obtenir une profondeur de pénétration suffisante pour éviter toute diffusion extérieure. Seules des raisons techniques ont imposé pour 1 'or une énergie de seulement $100 \mathrm{keV}$, ce qui ne permet pas d'éviter la diffusion vers 1 a surface. Les doses retenues, $5 \times 10^{16}$ à $2 \times 10^{17}$ ions. $\mathrm{Cm}^{-2}$ permettent d'obtenir un coefficient de remplissage $q$ (ou fraction volumique) de I'ordre de 0,2 - 0,3 dans la zone d'implantation. Les distances de pénétration $\mathrm{Rp}$ et la largeur à mi-hauteur $\Delta \mathrm{Rp}$ sont donnêes ci-dessous /4/:

\begin{tabular}{llllllll} 
& & \multicolumn{3}{c}{$\mathrm{Rp}$} & & & $\Delta \mathrm{Rp}$ \\
$\mathrm{Na}^{+}(600 \mathrm{keV})$ & 5 & 900 & $\AA$ & & 1 & 520 & $\AA$ \\
$\mathrm{Na}^{+}(750 \mathrm{keV})$ & 7 & 080 & $\AA$ & 1 & 560 & $\AA$ & $\AA$ \\
$\mathrm{Au}^{+}(100 \mathrm{keV})$ & & 260 & $\AA$ & & 110 & $\AA$
\end{tabular}

Les échantillons sont alors caractêrisés optiquement et structuralement, puis traités thermiquement sous un vide de $10^{-3}$ torr de $473 \mathrm{~K}$ à $1323 \mathrm{~K}$, en faisant des recuits par palier de $50^{\circ} \mathrm{C}$ pendant $15 \mathrm{~min}$. En effet il n'est plus constaté d'évolution cristalline déterminante au- 
delà de 15 min. ; sans doute la diffusion est alors suffisante pour assurer le quasi équilibre thermodynamique des phases en présence.

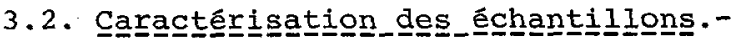

- La modification des propriétés optiques du matériau implanté est constatée par spectrométrie d'absorption : une bande large attribuée ordinairement à des colloìdes métalliques se développe dès $773 \mathrm{~K}$; à cette température les ions dispersés dans la zone d'implantation commencent à précipiter et, dans notre cas, à former des amas colloĩdaux métalliques de même nature que le métal implanté. Il est possible de suivre l'évolution de la précipitation en observant celle de cette bande d'absorption optique situêe dans la partie visible du spectre.

- La nature chimique, la structure cristalline et l'orientation de ces précipités par rapport à la matrice diélectrique sont déduites des observations faites par microscopie électronique à țransmission sur des échantillons préalablement amincis par bombardement ionique ; cet amincissement est dêlicat car il faut atteindre la zone implantée, sachant que les observations microscopiques valables concerneront une épaisseur de 3 à $4000 \AA$, ainsi toute la zone implantée est bien observable.

- Le profil de distribution des ions implantés est observé sur les spectres de rétrodiffusion de particules $\alpha$, d'analyse nucléaire, ou, après attaque ionique épluchant le matériau, par microsonde électronique. La méthode employée dépend de la nature du matériau, par exemple la proximité des masses de sodium et du magnésium rend impossible l'utilisation de la rétrodiffusion de particules $\alpha$ dans ce cas. Les tailles et les formes des amas observés sont déduites des images microscopiques. 3.3. Mesureseptigues.- Si les spectres d'absorption optique renseignent sur l'évolution des prếcipitês métaliliques, ils fournissent également le coefficient d'absorption en fonction de la longueur d'onde. Il est possible de connaître le coefficient $\alpha$ dans la gamme de 0,2 à 2,5 $\mu \mathrm{m}$, ainsi l'absorọtion sélective solaire peut être déterminêe. Les mesures sont faites à température ambiante sur les échantillons implantés et traités thermiquement de 873 à $1323 \mathrm{~K}$ pendant $15 \mathrm{~min}$.

L'implantation n'est pas toujours homogène, sauf s'il est possible d'utiliser un balayage électrostatique. Pour surmonter l'inhomogénéité possible d'implantation, les mesures optiques sont toujours faites dans la même zone pour chaque échantilion.

Les matériaux etant plus ou moins transparents, les mesures d'absorption sont en fait réalisées par transmission, aussi convient-il d'évaluer quelle partie de la lumière incidente est réfléchie pour déterminer la partie réellement absorbée. La réflectivité globale est mesurée en uti- 
lisant un système de sphères intégrantes équipant un spectrophotomètre Beckman Acta M IV. Des mesures de réflectivité directionnelle sont nécessaires pour obtenir les composantes Rs et $\mathrm{Rp}$ en fonction de l'angle d'incidence et de la longueur d'onde, un montage original a été réalisé (Fig. 2). Ainsi 1'évolution de la réflectivité peut être appréciée en fonction de l'évolution cristalline du matériau implanté.

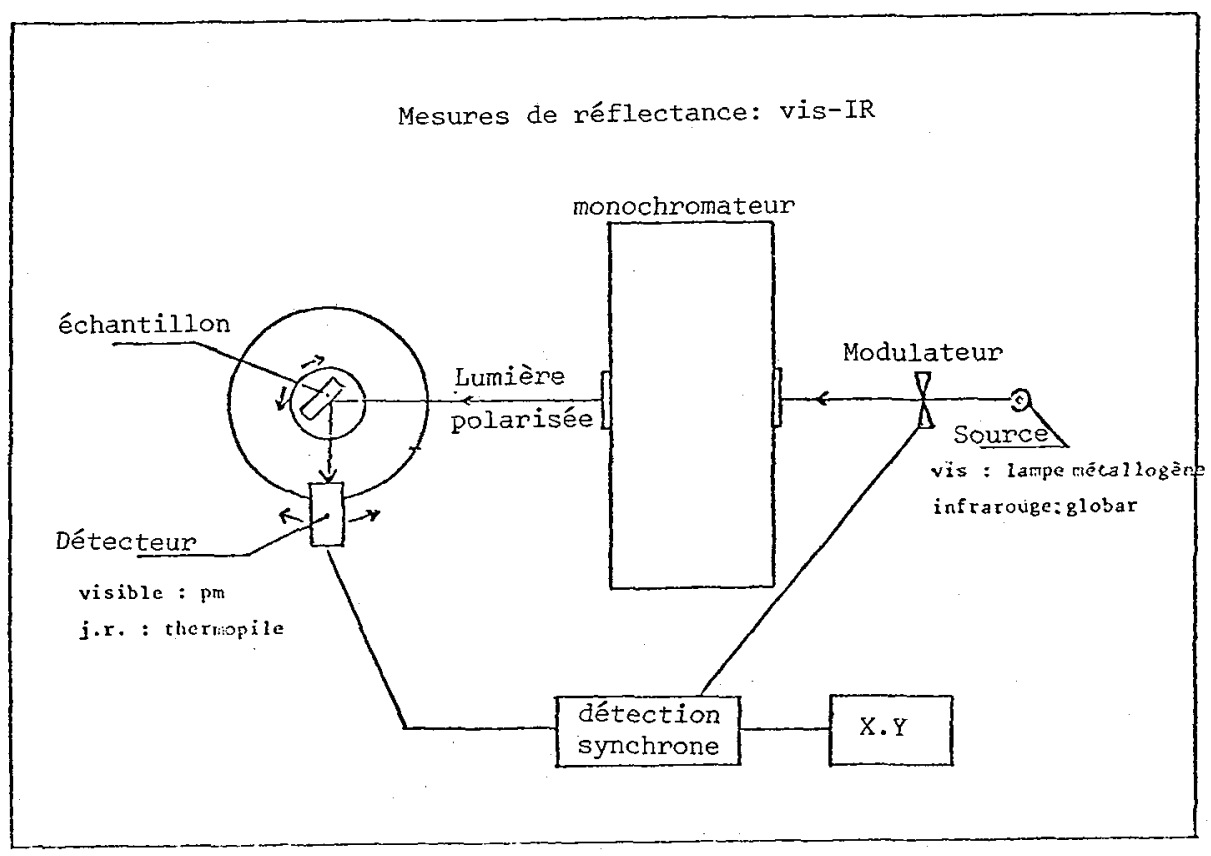

Fig. 2.- Schéma du montage optique pour mesurer la réflectivité spéculaire bidirectionnelle.

4. Caractérisation des inclusions métalliques.- Les premières études ont êté entreprises sur des monocristaux de Mgo implantếs avec du sodium, en raison de leurs propriétés optiques, du caractère puxement métallique du sodium et de la relative facilité technique d'implantation. Cependant, le profil de distribution du sodium est mal aisé à déterminer ; aussi les observations microscopiques ont dû supplêer à cette carence, la profondeur du champ d'observation est suffisante pour obtenir une appréciation du profil.

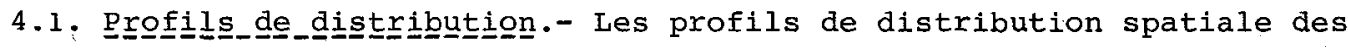
ions implantés peuvent être aisément calculés /4/. Cependant leur évolution en fonction du traitement thermique doit être appréciée en uti- 
lisant conjointement les techniques précédemment évoquées et la microscopie électronique à transmission. Par exemple pour Mgo : Au, la figure 3 montre le profil de distribution de l'or réalisé par rétrodiffusion de particules $\alpha$; en effet, le spectre en énergie des particules détectées est une image de la courbe de concentration de l'élément analysé. Ce spectre permet de déterminer non seulement l'allure du profil de distribution, mais également la profondeur de la zone implantée par rapport à la surface.

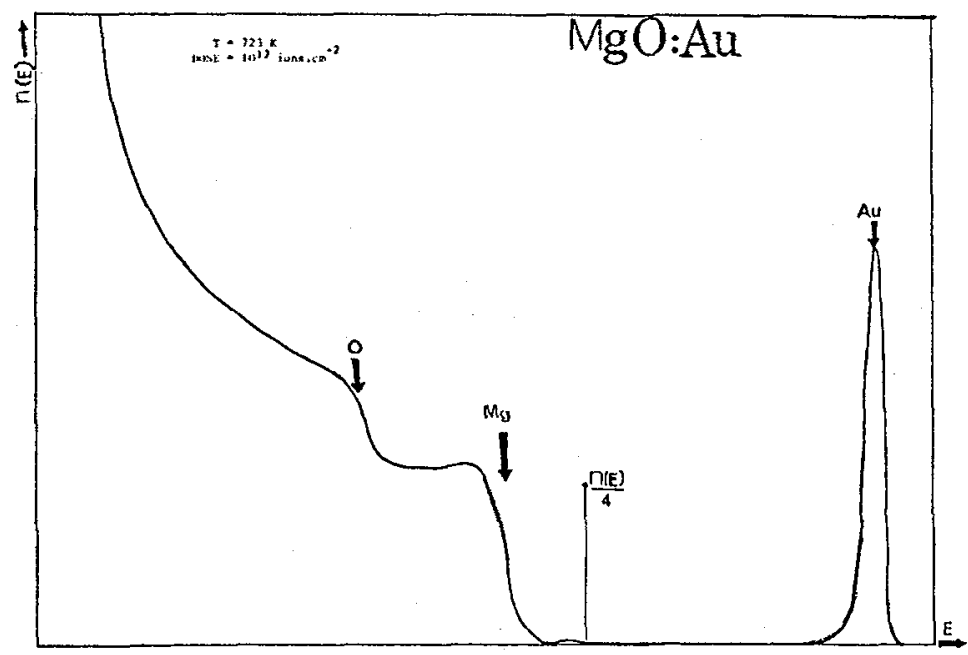

Fig. 3.- Profil de distribution de l'or implanté dans l'oxyde de magnêsium, estimé à l'aide de la rétrodiffusion de particules $\alpha$. On porte en fonction de l'énergie $E$; le nombre de coups d'énergie $E$.

Ainsi pour chaque êchantillon bien défini et à une température de recuit donnée, à partir du profil de distribution, il sera possible de calculer la fraction de volume q occupée en fonction de la profondeur considérée. Les observations microscopiques permettent de compléter cette information en précisant les tailles, la distribution des tailles et les formes des colloỉdes métalliques obtenus.

Par exemple, dans le cas de MgO : Na, la fraction de volume maximale sera respectivement de 0,$1 ; 0,2 ; 0,4$ selon la dose d'ions $\mathrm{Na}^{+}$ implantés $5 \times 10^{16} ; 10^{17} ; 2 \times 10^{17}$ ions. $\mathrm{cm}^{-2}$. On peut ausisi vérifier que l'aire des profils de distribution correspond bien à la dose choisie - en première approximation.

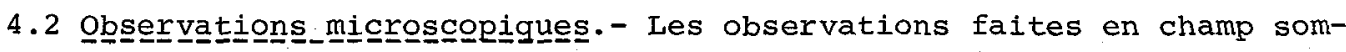
bre à partir des taches de diffraction supplémentaires par rapport à celles de Mgo et correspondant aux précipités vus en champ clair, permettent de déterminer l'identité physico-chimique de ces précipités (ici ce sont des colloïdes métalliques), leur orientation par rapport à la 
matrice, leurs tailles (et la distribution de ces tailles) et leurs formes gëométriques.

Pour ligo: Na, la phase précipitée est constituée de sodium métallique cubique centré orienté simplement par rapport à MgO. Une épitaxie simple en volume est observée :

$$
\text { (001) } \mathrm{Na} / /(001) \mathrm{MgO} \text { et }[100] \mathrm{Na} / /[100] \mathrm{MgO}
$$

Les colloïdes de sodium observés sont proches de la forme sphérique, aucune dimension n'étant privilégiée. Parfois des formes cuboïdales sont nettes, surtout pour les faibles tailles. Les diamètres moyens mesurés s'échelonnent de $120 \AA$ pour une température de recuit de $1073 \mathrm{~K}$, à $300 \AA$ pour une température de $1173 \mathrm{~K}$. La distribution des tailles devient plus importante lorsque là température de recuit crô̂t; en effet à partir des températures de recuit pour lesquelles l'observation microscopique a un sens (diamètre de $50 \mathrm{~A}$ ), c'est à dire $873 \mathrm{~K}$, la coalescence se développe et accentue la dispersion des tailles. Vers $1223 \mathrm{~K}$, quelques très gros collö̈des sont observés ( 2 à $3000 \AA$ ).

Pour MgO: Au, les premières observations ont montré que les colloïdes étaient petits et formés d'or, mais en raison de l'énergie insuffisante d'implantation et de la diffusion vers la surface, les conclusions sont peu utilisables. En implantant avec une énergie d'au moins $300 \mathrm{keV}$, des mesures utiles pourront être effectuées.

5. Propriétés optiques des cristaux implantés.- Si des mesures d'absorption ont été faites pour les deux types d'échantillons, les mesures de rëflexion n'ont été entreprises pour l'instant que sur les échantillons de $\mathrm{MgO}: \mathrm{Na}$.

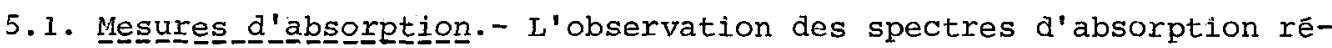
vèle la présence de deux bandes d'absorption dans la partie visible du spectre solaire. Ces deux bandes sont d'importance inégale corme le montrent les densités optiques (Fig. 4). Les positions de ces bandes évoluent en fonction de la température de traitement thermique.

Par exemple pour Mgo : Na, la bande de plus basse énergie a la plus haute densité optique, sa position évolue de 455 à $675 \mathrm{~nm}$ lorsque la température crồt de 673 à $1323 \mathrm{k}$ et la densitê optique atteint alors un maximum pour $1323 \mathrm{~K}$. Des estimations de tailles moyennes de colloídes métalliques sont faites à partir des mesures de fréquences des bandes d'absorption et de leurs largeurs à mi-hauteur.

Ces estimations dénotent une évolution des tailles en fonction de la. température de traitement thermique : le diamètre moyen varie de $70 \mathrm{~A}$ $(873 \mathrm{~K})$ à $150 \AA$ A $(1323 \mathrm{~K})$. Les observations microscopiques vont dans le même sens que ces estimations. Ainsi se révèle une évolution corrélée 
de la position des pics d'absorption et des tailles des.inclusions métalliques de sodium.

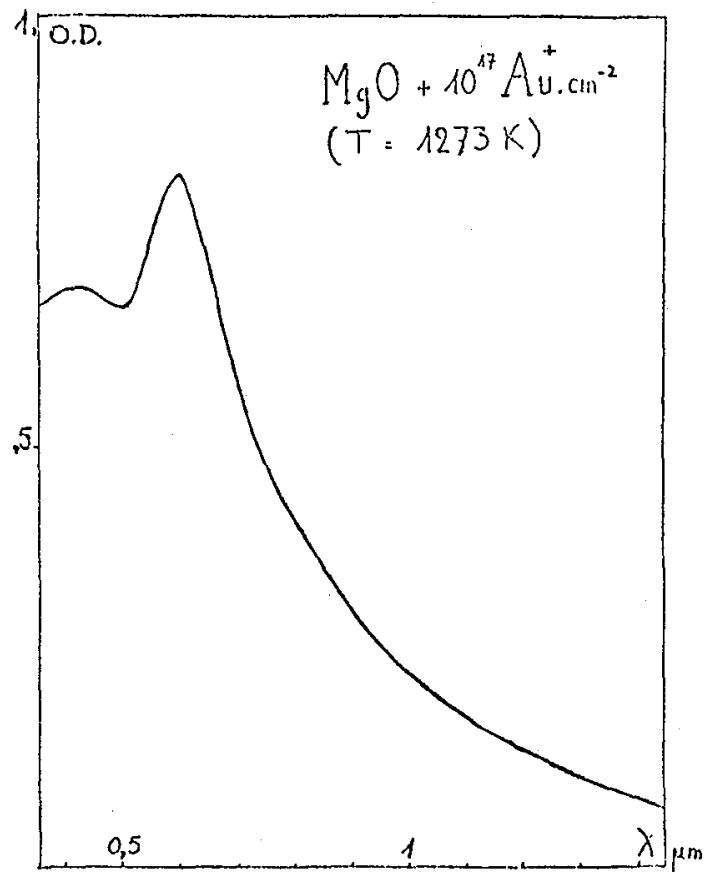

Fig. 4.: $2_{2}$ Spectre d'absorption d'un échantillon de MgO : Au (dose $=10^{17}$ ions. $\mathrm{cm}^{-2}, \mathrm{~T}$ recuit $\left.=1273 \mathrm{~K}\right)$. On remarque deux bandes situées à 420 et $600 \mathrm{~nm}$.

La présence de ces deux bandes d'absorption est en bon accord avec les calculs faits en utilisant la théorie de Maxwell-Garnett appliquée à des inclusions métalliques enrobées dans une matrice diêlectrique /2/. Ces deux bandes correspondent à la bande de conduction optique (d'ênergie la plus basse) et à celle de résonnance de plasma (de plus forte énergie), leurs fréquences calculées sont déduites de la relation :

$$
\frac{\tilde{\varepsilon}(\omega)-n_{0}^{2}}{\tilde{\varepsilon}(\omega)+2 n_{0}^{2}}=q \frac{\tilde{\varepsilon}_{m}(\omega)-n_{o}^{2}}{\tilde{\varepsilon}_{m}(\omega)+2 n_{0}}
$$

où $\mathrm{n}_{0}$ est 1 'indice de réfraction réel de la matrice MgO $(=1,73)$ $\tilde{\varepsilon}_{\mathrm{m}}(\omega)=\varepsilon_{1 \mathrm{~m}}(\omega)+i \varepsilon_{2 \mathrm{~m}}(\omega)$, la constante diélectrique complexe du mêtal $\tilde{\varepsilon}(\omega)=\varepsilon_{1}(\omega)+i \varepsilon_{2}(\omega)$, celle du matériau composite (MgO: Na) $q$ est le coefficient de remplissage.

Les valeurs de $\varepsilon_{1 \mathrm{~m}}$ et $\varepsilon_{2 \mathrm{~m}}$ sont celles du sodium massif, en première approximation, les valeurs correspondant au sodium granulaire étant mal connues. 
5.2 Mesures_de_réflex $4 \mathrm{~N}$ utilisés sont légèrement nuageux et diffusent quelque peu. Cet aspect est dû à de très fines bulles (d'hydrogène) dispersées dans le matériau. En dépit de cette diffusion les mesures faites sur les échantillons implantés et recuit indiquent une faible valeur de la réflectivité entre 0,3 et $2,5 \mu \mathrm{m}$, de 1 'ordre de 0,2 - 0,3. Ainsi l'absorption précédemment mesurée est-elle importante dans la partie visible du spectre solaire et confère aux matériaux transparents étudiés des propriétés d'absorption solaire assez intéressantes.

5.3 Mesures_de_réflexion bidirectionnelle.- Ces mesures ont eté entreprises pour compléter les mesures précédentes ; en effet grâce au montage réalisê, il est possible de mesurer pour un angle d'incidence donné la valeur des composantes polarisées parallèle Rp et perpendiculaire Rs de la réflectivité spēculaire en fonction de la longueur d'onde, mais ceci uniquement dans la partie visible du spectre solaire, pour le moment. L'angle d'incidence peut varier de 0 (incidence normale) à $60^{\circ}$; au-delà, les mesures sont peu significatives en raison de la géométrie du faisceau, de l'ouverture de fente nécessaire pour mesurer Rp (proximitê de l'incidence de Brewster) et de la surface implantée trop faible $\left(1 \mathrm{~cm}^{2}\right)$.

Dans le cas de Mgo : Na, si l'allure des courbes de réflectivité Rs et Rp est habituelle pour les premiers stades du traitement thermique, on constate dès $873 \mathrm{~K}$ et pour toutes les incidences observées, $1^{\prime}$ apparition d'une modulation, relativement modeste, des coefficients de réflectivité en fonction de la longueur d'onde. La figure 5 correspond à la réflectivité Rs d'un échantillon de Mgo : Na recuit à $1323 \mathrm{~K}$ et éclairé sous une incidence de $45^{\circ}$; en fait la courbe représente le rapport de la réflectivité Rs de l'échantilion implanté à celle de l' échantilion pur. L'allure de cette courbe suggère un phénomène interférentiel.

or on constate qu'à partir de l'apparition de cette modulation (recuit $\mathrm{T}=873 \mathrm{~K}$ ), les positions des maximas de ces franges évoluent jusqu'à un recuit $T=1123 \mathrm{~K}$. A partir de cette température les franges sont fixées, seule leur amplitude peut légèrement différer.

Cette évolution est à rapprocher de celle des inclusions métalliques de sodium : I'évolution des tailles est observable optiquement et microscopiquement dès $873 \mathrm{~K}$, cette évolution se manifeste jusqu'à $1123 \mathrm{~K}$ (taille moyenne voisinant $300 \AA$ ), puis se stabilise nettement, même si les tailles augmentent encore un peu.

Aussi est-on tenté d'attribuer l'allure des courbes de réflectivité spéculaire bidirectionnelle à la présence des colloídes métalliques dans la zone implantêe : des franges d'interférence se produiraient dans 
cette zone.

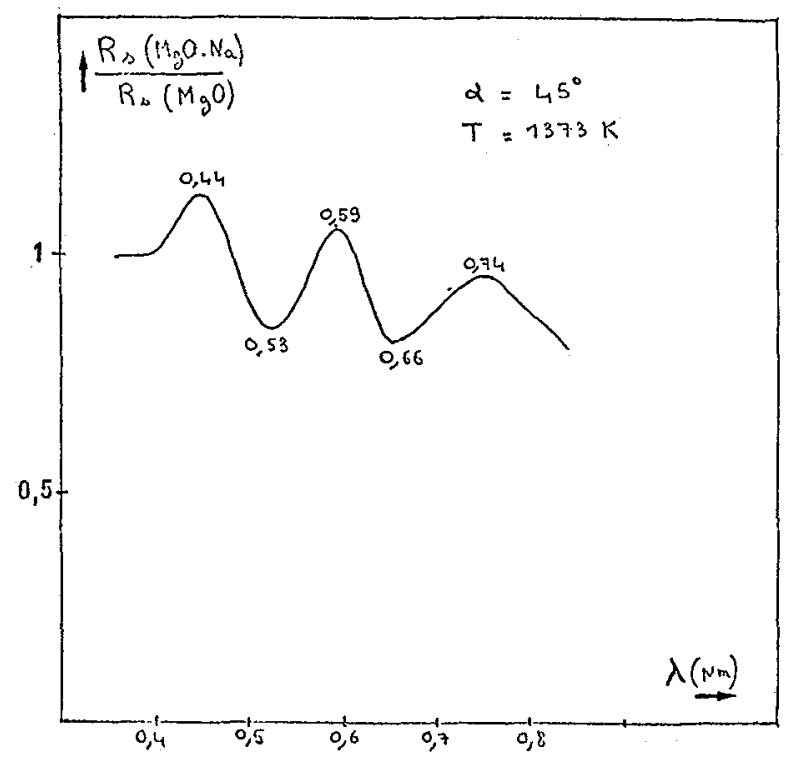

Fig. 5.- Comparaison des coefficients de réflectivité Rs de l'oxyde de magnésium, implanté en sodium et pur (après un recuit à $1323 \mathrm{~K}$ ), mesurés sous incidence de $45^{\circ}$. Des franges d'interférences dues à la couche implantée serablent mises en évidence.

6. Premières interprétations.- Elles concernent essentiellement les échantillons de MgO : Na pour lesquels des mesures significatives ont pu être réalisées, en utilisant plusieurs techniques fournissant des infornations complémentaires.

- Les observations microscopiques confirment le caractère de cermet présenté par ce matêriau transparent implanté. Après traitement thermique bien choisi, les tailles des inclusions métalliques et leur profil de distribution peuvent être évalués et demeurent stables dans le temps et après retour à la température ambiante. Les mesures optiques montrent' le caractère absorbant de ce matériau dans la partie visible du spectre solaire et sa faible réflectivité dans l'infra-rouge, cette qualité est essentielle pour un matériau sêlectif. Ainsi par implantation, nous avons obtenu un matériau absorbant sélectif, transparent et réfractaire, sans évolution constatée à des températures pouvant aller jusqu'à $1273 \mathrm{~K}$ après traitement thermique de $15 \mathrm{~min}$ à même température.

- Un essai d'interprétation des franges observées lors des mesures de réflectivité bidirectionnelle, est tenté en utilisant un programme de calcul expérimenté avec succès dans le cas du chrome noir /5/. Les premiers résultats du calcul montrent que la zone implantée peut donner lieu à des franges a'interférence ayant une allure comparable à celles 
observées. Cependant, cette approche doit être complétée en utilisant une meilleure connaissance du profil de distribution des inclusions après recuit. En effet dans le modèle développé, le matériau est considéré comme un empilement de couches minces, chaque couche est caractérisée par une matrice équivalente dont les paramètres essentiels sont calculés à partir de la constante diélectrique complexe du matériau et du coefficient de remplissage $q$, qui varie selon la couche considérée en fonction de la profondeur. Actuellement notre connaissance de ce facteur $q \mathrm{n}$ 'est pas assez précise en raison des difficultés de mesure du profil par rétrodiffusion de particules $\alpha$.

7. Conclusion.- L'étude commencée avec MgO : Au devrait conduire à une meilleure comparaison des résultats expérimentaux et des modèles proposés utilisant les théories développées et basées sur l'électrostatique (Maxwell-Garnett) ou sur l'électromagnétisme (Lord Rayleigh). En effet les constantes optiques sont mieux connues et la caractérisation semble plus aisée.

Mais c'est essentiellement la caractérisation soignée du matériau et les mesures optiques d'absorption et de réflexion dans toute l'étendue du spectre solaire qui permettront de mieux comprendre les propriétés sélectives présentées par le cermet ainsi mis en oeuvre, ce qui peut être important pour la réalisation pratique d'un cermet utilisable dans la gamme de températures de 300 à $500^{\circ} \mathrm{C}$.

Remerciements.- Nous tenons à exprimer notre gratitude pour l'accueil dans leur laboratoire et pour les discussions fructueuses, d'une part à Monsieur le professeur F. Abeles, Directeur du Laboratoire d'optique des Solides de l'Université Pierre et Marie Curie (Paris VI) et à ses collaborateurs J. Lafait, J.M. Behaghel et S. Berthier, d'autre part à Monsieur Jean Spitz, Chef du Département de Métallurgie au C.E.N. Grenoble et à ses collaborateurs, Messieurs G. Blandenet, I. Ritchie et J. Valignat. 


\section{Bibliographie}

/1/ Treilleux, M. et al., Phys. Status Solidi

(a) 48 (1978) -425

Trei 11 eux, M. and Chassagne, G., J. de Physique Lett. 40 (1979) - L.283 et 40 - L. 161

Guermazi, M. et al., Rad. Effects, 37 (1978) - 99

Guermazi,M. et al., Mat. Res. Bull.,

$15(1980)-147$

Picraux S.T., in "Site Characterization and Aggregation of Implanted Atoms in Material" (Plenum Pub.Co) (1980) $307-325$

12/ Marichy,G., Chassagne,G. and Durand,D., Phys. Status Solidi (b) 92 (1979) - 221

/3/ Lampert,C.M., Sol.Energy Mater. 2 (1980) - 1

/4/ Dearnaley, G. and al., in "Ion implantation", (North Holland Pub. Co.) (19.73) 756

15/ Berthier,s., Thèse 3ème Cycle - Université Paris VI (1979)

Berthier, S. et Lafait,J., J. Physique Colloq. (cette conférence) 